\title{
¿Todos los caminos llevan a Washington? Trayectorias de América Latina hacia un Acuerdo de Libre Comercio con los Estados Unidos (1990-2015)
}

\section{All roads lead to Washington? The paths of Latin American countries towards a Free Trade Agreement with the United States (1990-2015)}

\author{
Rodrigo Cuevas Ossandón ${ }^{1}$ \\ Universidad de Valparaíso, Chile \\ JAQUELIN MORILLO ReMESNITZKY ${ }^{2}$ \\ Investigadora Independiente, Chile
}

\begin{abstract}
RESUMEN Este trabajo se enfoca en la entrada en vigor de un acuerdo comercial entre un país latinoamericano y Estados Unidos. Para ello, el objetivo propuesto es estudiar las trayectorias hacia la ocurrencia de una vinculación de este tipo con la principal potencia del mundo en el periodo 1990-2015 a partir de tres grupos de variables: reformas de mercado, geopolítica de los acuerdos y vinculación comercial, siendo las primeras las más relevantes en dar cuenta de este proceso. El enfoque que se empleó es un Análisis de Eventos Históricos, por medio de regresión de Cox, a fin de identificar determinantes que expliquen dicho proceso.
\end{abstract}

PALABRAS CLAVE Regionalismo; liberalización económica; reformas de mercado; cambio institucional; análisis de sobrevivencia; América Latina.

ABSTRACT This paper discusses the entry into force of trade agreements between Latin American countries and the United States. The objective is to study paths towards the implementation of a free trade agreement with the world's

1. Rodrigo Cuevas: Doctor en Ciencias Sociales, Universidad de Chile. Profesor Escuela de Administración Pública, Universidad de Valparaíso, Campus Santiago. E-mail: rodrigo.cuevas@uv.cl.

2. Jaquelin Morillo Remesnitzky: Magíster en Estrategia Internacional y Política Comercial. Investigadora Independiente. E-mail: jaquelin.morillo@gmail.com. 
greatest power in the period 1990-2015, considering three groups of variables: market reforms, geopolitics of trade agreements and commercial links with the world. The results suggest that market reforms have the greatest explanatory impact on the principal outcome of this study. The research technique used was Event History Analysis, implemented through Cox regression, in order to identify determinants that explain the process. Although this approach has been widely used in the field of international relations, mainly in the study of conflicts or crises, it is novel in the analysis of the entry into force of trade agreements.

KEYWORDS Regionalism; Economic Liberalization; Market Reforms; Institutional Change; Survival Analysis; Latin America

\section{Introducción}

En el presente artículo se analiza la vinculación comercial de América Latina con Estados Unidos, específicamente, qué llevó a que los países de la región comenzaran a implementar un acuerdo comercial que los vinculara con la principal potencia del mundo. En este trabajo se estudia qué variables fueron las más relevantes a la hora de explicar por qué un país de la región tomó la decisión descrita. Para ello, se empleó un enfoque de Análisis de Eventos Históricos (Event History Analysis o EHA), implementada mediante la aplicación de regresiones de Cox, sobre una base de datos que consta de 520 observaciones, correspondiente a 21 países entre 1990 y $2015^{3}$, que son los siguientes: Argentina, Bolivia, Brasil, Chile, Colombia, Costa Rica, Cuba, República Dominicana, Ecuador, El Salvador, Guatemala, Honduras, Jamaica, México, Nicaragua, Panamá, Paraguay, Perú, Uruguay y Venezuela. Estos fueron agrupados en dos subregiones: los países de América del Sur y los de América Central, México y los tres principales estados del Caribe, que son Cuba, República Dominicana y Jamaica.

En el artículo, se sostiene que la introducción e implementación de procesos de reformas económicas, orientadas a la liberalización, aparecen como factores que aceleran la probabilidad de la entrada en vigor de un acuerdo comercial con los Estados Unidos. Estas serían más importantes que otro tipo de determinantes que favorecen una integración comercial como la cercanía geográfica y la importancia del tamaño del mercado de la principal potencia del mundo en la región.

3. BECK y KATZ (1995); PICKUP (2015). 
El presente artículo se organiza en las siguientes secciones. En primer lugar, se expone el problema de investigación, prestando especial importancia a los principales argumentos que dan cuenta del fenómeno de los acuerdos de libre comercio, planteando que, para el caso del estudio analizado en este artículo, es más conveniente enfocarse en las variables ligadas a las reformas de mercado y a la apertura comercial.

A continuación, se define el objeto de estudio, que es el estudio específico de los acuerdos de libre comercio de los países de la región con EE.UU., exponiéndose las implicancias de contraer compromisos comerciales con la principal potencia del mundo. El registro de la entrada en vigor de un acuerdo con los Estados Unidos, el evento a analizar, se definió en base a la información proporcionada por la Organización de Estados Americanos ${ }^{4}$. Esta primera sección concluye con la pregunta de investigación, sus hipótesis asociadas y la pertinencia del uso de EHA respecto a otras formas de modelación.

Luego, se describe la variable dependiente, definida como la puesta en vigor de un acuerdo con los Estados Unidos en el período de tiempo observado, 1990-2015. Asimismo, se precisan las co-variables que explicarían la propensión a implementar un acuerdo con Estados Unidos y se plantea el diseño metodológico, describiendo las características del EHA, en general, y del uso del modelo propuesto por David Cox ${ }^{5}$, en particular.

La siguiente sección presenta los resultados obtenidos por medio de la aplicación de esta técnica e introduce las correcciones pertinentes respondiendo a la estructura de datos y al cumplimiento de supuestos de la estimación realizada. Finalmente, se presentan los hallazgos y principales conclusiones obtenidas.

\section{Problematización}

El primer acuerdo de libre comercio entre un país de América Latina y Estados Unidos entró en vigor en el año 1994, que fue la concurrencia de México en el Tratado de Libre Comercio de América del Norte (NAFTA, por sus siglas en inglés). En el periodo estudiado, los últimos casos corresponden a Colombia y Panamá en el año 2012. Sin embargo, entre 1994 y 2012, surgieron diversas negociaciones entre los países latinoamericanos y Estados Unidos, algunas de ellas culminaron en compromisos exitosos como, por ejemplo, el caso del acuerdo Chile-Estados Unidos, el cual entra en vigor en 2004. Otras se vieron truncadas por razones variadas, entre ellas, un cambio de orientación de la política interna y las dificultades propias de los procesos negociadores, como sucedió con la iniciativa Ecuador-Estados Unidos en el año 2007.

4. Al respecto, ver Sistema de Información de Comercio Exterior (SICE), http://www.sice.oas.org/ ctyindex/USA/USAagreements_s.asp, acceso 10 de Julio de 2019.

5. COX (1972). 
En términos de una inserción como objeto de estudio, los acuerdos comerciales suelen ser considerados como variables explicativa de una diversidad de fenómenos, tales como la disminución de conflictos, procesos de reformas económicas, aumento del comercio, la democracia, entre otras materias, más que ser una variable dependiente ${ }^{6}$. En ese sentido, se busca analizar estos tratados como una variable a explicar.

Los acuerdos de libre comercio (ALC) constituyen una de las vías por medio de las cuales los países definen su inserción comercial bilateral siendo las otras de tipo unilateral o multilateral, modalidades que no se aplican de manera excluyente. Una de las características específicas de los acuerdos de libre comercio es que los beneficios sólo son aplicables a las partes involucradas en ese acuerdo ${ }^{7}$, a diferencia de acuerdos multilaterales, como son los acuerdos negociados en el marco de la Organización Mundial del Comercio (WTO) o de medidas que toma un gobierno de manera autónoma, como una rebaja de aranceles a las importaciones ${ }^{8}$. En ese sentido, se les ha considerado como una herramienta que profundiza procesos de integración ${ }^{9}$.

También se les ha asignado un papel de gran importancia en evitar los conflictos armados, donde el principal argumento sugiere que los países comercian más entre sí reducen el riesgo de un conflicto militar, condición que adquiere más relevancia cuando se trata de países democráticos ${ }^{10}$.

Un tercer argumento es que la liberalización del comercio sería una variable que permitiría mayores niveles de democracia, fundamentalmente, en la reticencia por parte de las dictaduras a usar herramientas como un FTA, debido a la pérdida de sus espacios de discrecionalidad al comprometer la reducción de espacios de este tipo a la aplicación de sus políticas ${ }^{11}$ y más aún, que los países con mayores niveles de democracia llevaría a que los compromisos contenidos en los acuerdos sean de mayor profundidad $^{12}$.

Respecto al papel que cumplen los acuerdos de libre comercio en las reformas de mercado, los ALC constituyen una certificación de la credibilidad por parte del país suscriptor, en que mantendrá o profundizará su orientación pro-mercado ${ }^{13}$, en especial cuando está involucrada una relación del tipo Norte-Sur; es decir, en la situación

6. WTO (2011); RAVENHILL (2011).

7. ALLE y ELSIG (2016).

8. WTO (2011).

9. WTO (2011); RAVENHILL (2011).

10. GARTZKE (2007); RUGGIE (1982); POLACHEK (1997); BALDWIN (1980); BLISS y RUSSET (1998); GARTZKE (1998); MACMILLAN (1997).

11. MANSFIELD et al. (2008); MANSFIELD y MILNER (2012).

12. HÜHNE et al. (2015).

13. WTO (2011); BACCINI y URPELAINEN (2014); ALLE y ELSIG (2016); MANSFIELD y MILNER (2012); RODRIK (2000). 
en que un país en desarrollo tiene como contraparte a uno que ya superó esa etapa ${ }^{14}$. En ese caso, los ALC contribuirían a apuntalar el liderazgo político durante la implementación de las reformas de mercado $^{15} \mathrm{o}$ en el fortalecimiento de la orientación pro-mercado de las mismas en el mediano plazo $^{16}$. Finalmente, un acuerdo tendería a mayores grados de profundización en su orientación liberalizadora si predomina el comercio de bienes intermedios que finales ${ }^{17}$, que da cuenta de economías más integradas en términos de su industria, condición que aplicaría a casos como el de México y Estados Unidos.

El auge de la entrada en vigor de acuerdos de libre comercio en América Latina ocurre en un contexto como el de la década de 1990, en que se reinauguran democracias y se inician reformas de reformas de mercado, con excepciones como Chile, que ya las había iniciado en $1974^{18}$, con una diversidad amplia de resultados nacionales en estas dimensiones. Entre los países latinoamericanos estudiados se observa una importante diversidad respecto de sus características e indicadores económicos y políticos. Sus economías de mercado presentan un desempeño dispar que oscila entre los 4.1 y 8.6 puntos del Índice de Transformación Bertlesmann ${ }^{19}$. En lo que respecta a democracia, son considerados mayormente como democracias plenas, pero se manifiestan casos en que esa condición es discutible, como el de Honduras ${ }^{20}$. Asimismo, de acuerdo con su adhesión a un modelo de libertad económica, en general, son países clasificados como "moderadamente libres" por el Índice de Libertad Económica (IEF), aunque con excepciones como Nicaragua. El Índice de Libertad Económica, que desarrolla el Instituto Heritage y el diario Wall Street Journal, mide una serie de indicadores relacionados con la libertad económica. Cada país es medido en una escala de 1 a 100 y a partir de sus resultados se clasifican de la siguiente manera: "libre" (100-80); "mayoritariamente libre" (79,9-70); "moderadamente libre" (69.9-60); "mayoritariamente no libre" (59.9-50); "represivo" (49.9-0)

\footnotetext{
14. ALLE y ELSIG (2016).

15. BACCINI Y URPELAINEN (2014).

16. ALLE y ELSIG (2016); CUEVAS (2019).
}

17. BACCINI et al. (2018).

18. STOKES (2001); TEICHMAN (2011); FFRENCH-DAVIS (2005).

19. Este indicador de desempeño de una economía de mercado es tomado a partir de los resultados de la Índice de Transformación de la Fundación Bertlesmann, con una escala entre 1 y 10 puntos, donde valores superiores a 7 indican un desempeño positivo en sus economías de mercado. Al respecto ver, https://www.bti-project.org/en/index/status-index/, acceso, 11 de Julio de 2017.

20. De acuerdo con el Î́ndice Polity IV, un país es considerado democrático si obtiene un resultado sobre 6 puntos. MARSHALL (2013).

21. Ver anexo 1. Mayor detalle ver en Index of Economic Freedom, http://www.heritage.org/index/, acceso, 11 de Julio de 2017. 


\section{i) Por qué estudiar los acuerdos comerciales de América Latina y EE.UU.}

La opción de centrarnos en el caso específico de Estados Unidos tiene que ver con lo que significa en América Latina una vinculación con la principal potencia del mundo, tanto en términos comerciales como políticos, y fundamentalmente, comprender qué llevó finalmente a un país de la región a firmar un ALC con Estados Unidos (EE.UU.). Las prioridades estadounidenses se condensaron en una iniciativa de corte hemisférico, la cual se consolidó en la propuesta de la Alianza de Libre Comercio de las Américas (ALCA). Tras grandes debates en numerosos países de la región, en la Cumbre de las Américas realizada en Mar del Plata en el año 2005, el ALCA se vio coartado, en gran medida, por movilizaciones sociales e incoherencia con los modelos de desarrollo latinoamericanos, lo que modificó el terreno de juego en la arena comercial entre EE.UU. y la región.

Como corolario, la posición de la región se agrietó ideológicamente ${ }^{22}$. Mientras que para algunos países la propuesta estadounidense representaba una oportunidad para dinamizar los propios modelos de desarrollo, aumentando los niveles de intercambio no sólo en el ámbito económico sino también cultural y de valores comparti$\operatorname{dos}^{23}$, para otros la relación comercial con la potencia del norte implicaría el primer eslabón de una cadena de dependencia ante el imperialismo norteamericano ${ }^{24}$.

Acto seguido, la política comercial estadounidense tomó un importante giro, optando por una estrategia bilateral en materia de acercamiento comercial con América Latina. Tras 12 años de la caída del ALCA, 11 países latinoamericanos y caribeños firmaron acuerdos de libre comercio con Estados Unidos.

A la fecha, existen seis acuerdos que vinculan comercialmente a países de la región con la principal potencia del mundo. Cuatro de estos son de corte bilateral (Chile, Panamá, Colombia y Perú), uno de ellos es multilateral (CAFTA) e involucra a Costa

22. "El ALCA se convirtió en un debate ideológico y se dice que el que está en contra es de izquierda y el que está a favor es de derecha". Lula Da Silva, durante la Organización Mundial de Comercio el 23 de noviembre de 2005. Fuente: "10 frases contra el ALCA", Telesur, publicado el 4 noviembre 2016, consultado en http://www.telesurtv.net/news/10-frases-contra-el-ALCA-20151102-0043. html el 21 de agosto de 2017.

23. "Esto significa la generación de más puestos de trabajos, más empleo y más oportunidades. También significa la posibilidad de poder tener un intercambio mayor no sólo en el ámbito económico sino también cultural y de valores compartidos con Estados Unidos". Ex Presidente Ricardo Lagos. Fuente: "Presidente Lagos anunció tratado de libre comercio con EE.UU., Emol, publicado el 29 de noviembre de 2000, consultado en http://www.emol.com/noticias/todas/2000/11/29/39730/presidente-lagos-anuncio-tratado-de-libre-comercio-con-eeuu.html el 21 de agosto de 2017.

24. "¿Quién enterró al Alca? Los pueblos de América enterramos al Alca, hoy, aquí en Mar del Plata". Hugo Chávez, discurso en Mar de Plata (Argentina) 4 de noviembre de 2005. Fuente: "10 frases contra el ALCA", Telesur, publicado el 4 noviembre 2016, consultado en http://www.telesurtv.net/ news/10-frases-contra-el-ALCA-20151102-0043.html el 21 de agosto de 2017. 
Rica, El Salvador, Guatemala, Honduras, Nicaragua y República Dominicana, mientras que México se vincula con Estados Unidos junto a Canadá por medio del NAF$\mathrm{TA}^{25}$. Para efectos del artículo, no se considera el proceso de re-negociación de este acuerdo iniciado bajo la presidencia de Donald Trump.

Una diferencia substancial que vale la pena destacar es el tiempo que media entre la firma del tratado y su respectiva puesta en vigor. Si bien esta característica tiene que ver con una doble dimensión, en la cual se involucran aspectos como la tramitación legislativa en los EE.UU. y su contraparte latinoamericana, el tiempo transcurrido desde la firma de un tratado hasta que este entra en vigor es bastante extenso, dado que el promedio es de casi tres años (33 meses). En los últimos casos, el proceso de ratificación se extendió a casi 5 años (Tabla 1 ).

Al mismo tiempo, las singularidades del Congreso estadounidense respecto de sus símiles latinoamericanos hacen difícil la comparación, debido a que tiene amplias atribuciones en materia de comercio exterior, donde puede intervenir en la propia negociación. Al mismo tiempo, su autonomía respecto al poder ejecutivo es bastante inusual en un régimen presidencial, lo que implica que cada senador o representante tenga un amplio margen de acción ${ }^{26}$. No obstante, la información presentada en la Tabla 1 nos sugiere que el proceso de entrada en vigor ha tendido a complejizarse a medida que más países han adquirido este tipo de vinculación con la principal potencia del mundo.

25. Mayor detalle de esta información se encuentre disponible en el Sistema de Información de Comercio Exterior de la Organización de Estados Americanos, http://www.sice.oas.org/ctyindex/ USA/USAagreements_s.asp , último acceso 15 de Noviembre de 2020.

26. BOX-STEFFENSMEIER et al. (1997); KAROL (2007). 
Tabla1: Entrada en vigor de Acuerdos comerciales de Estados Unidos con países de América Latina (1990-2015).

\begin{tabular}{|c|c|c|c|c|}
\hline \multirow[t]{2}{*}{ País } & \multirow{2}{*}{$\begin{array}{l}\text { Tipo de } \\
\text { Acuerdo }\end{array}$} & \multicolumn{3}{|c|}{ Diferencias entre fechas de firma y vigor } \\
\hline & & Firma & Vigor & $\begin{array}{c}\text { Tiempo } \\
\text { (en meses aproxi- } \\
\text { mados) }\end{array}$ \\
\hline México & $\begin{array}{c}\text { Plurilateral } \\
\text { (NAFTA) }\end{array}$ & $17 / 12 / 1992$ & 01/01/1994 & 12 \\
\hline Chile & Bilateral & $01 / 06 / 2003$ & $01 / 01 / 2004$ & 6 \\
\hline El Salvador & $\begin{array}{c}\text { Plurilateral } \\
\text { (CAFTA) }\end{array}$ & $05 / 08 / 2004$ & $01 / 03 / 2004$ & 18 \\
\hline Honduras & $\begin{array}{c}\text { Plurilateral } \\
\text { (CAFTA) }\end{array}$ & $05 / 08 / 2004$ & $01 / 04 / 2006$ & 19 \\
\hline Nicaragua & $\begin{array}{c}\text { Plurilateral } \\
\text { (CAFTA) }\end{array}$ & $05 / 08 / 2004$ & $01 / 04 / 2006$ & 19 \\
\hline Guatemala & $\begin{array}{c}\text { Plurilateral } \\
\text { (CAFTA) }\end{array}$ & $05 / 08 / 2004$ & $30 / 06 / 2006$ & 22 \\
\hline República Dominicana & $\begin{array}{c}\text { Plurilateral } \\
\text { (CAFTA) }\end{array}$ & $05 / 08 / 2004$ & $01 / 03 / 2007$ & 30 \\
\hline Perú & Bilateral & $12 / 04 / 2006$ & $01 / 02 / 2009$ & 33 \\
\hline Costa Rica & Plurilateral & 05/08/2004 & 23/12/2009 & 64 \\
\hline Colombia & Bilateral & $22 / 11 / 2006$ & $15 / 05 / 2012$ & 65 \\
\hline Panamá & Bilateral & $28 / 06 / 2007$ & $31 / 10 / 2012$ & 64 \\
\hline
\end{tabular}

Fuente: Elaboración propia en base a Sistema Integrado de Comercio Exterior (SICE).

Otro aspecto destacable de EE.UU. como contraparte de un acuerdo comercial es su condición de principal potencia económica y militar del mundo. Por tanto, el país que se involucra en un acuerdo comercial con los Estados Unidos, generalmente, se encuentra en condiciones de asimetría estratégica que se evidencia en diversas dimensiones. Por ejemplo, en 2016, su producto interno bruto (PIB) casi duplicaba el de China, era casi cinco veces el tamaño de la economía alemana, y casi dos tercios mayor que el de América Latina y el Caribe en su conjunto (Tabla 2). Estas diferencias se acentúan aún más en el plano militar, donde su gasto es tres veces mayor que el de China y casi diez la totalidad de los países de América Latina y el Caribe ${ }^{27}$. 
Tabla 2: Importancia de países seleccionados y Latinoamérica respecto a EE.UU. (\%).

\begin{tabular}{|c|c|c|c|c|c|}
\hline País & PIB & $\begin{array}{c}\text { PIB per } \\
\text { Cápita }\end{array}$ & Población & $\begin{array}{c}\text { Gasto } \\
\text { militar }\end{array}$ & $\begin{array}{c}\text { Gasto } \\
\text { militar per } \\
\text { cápita }\end{array}$ \\
\hline China & 0.54 & 0.13 & 4.27 & 0.36 & 0.08 \\
\hline Alemania & 0.22 & 0.88 & 0.25 & 0.07 & 0.26 \\
\hline Japón & 0.36 & 0.91 & 0.39 & 0.07 & 0.17 \\
\hline India & 0.14 & 0.03 & 4.08 & 0.09 & 0.02 \\
\hline Brasil & 0.14 & 0.22 & 0.65 & 0.04 & 0.06 \\
\hline México & 0.07 & 0.18 & 0.40 & 0.01 & 0.03 \\
\hline América Latina y el Caribe & 0.36 & 0.18 & 1.97 & 0.11 & 0.06 \\
\hline
\end{tabular}

Fuente: Elaboración propia en base a SIPRI, 2017 y World Development Indicators, 2017.

Un segundo elemento para entender la relevancia de estudiar la entrada en vigor de compromisos comerciales con EE.UU. radica en las complejidades de los procesos de implementación y las reformas estipuladas en los textos de los acuerdos. Por lo general, estas afectan a áreas de gran importancia en el funcionamiento de las economías de los países latinoamericanos, en especial, en el plano regulatorio, propiedad intelectual, compras públicas, inversiones o, incluso, en la normativa laboral ${ }^{28}$. En este sentido, la entrada en vigor de un acuerdo con Estados Unidos implica una modificación que afecta de manera integral la estructura económica y productiva a nivel interno de los países suscriptores ${ }^{29}$. Considerando este argumento, hemos optado por enfocarnos en el estudio de la entrada en vigor de dichos compromisos y no en su firma dado que, por las características legales de estos acuerdos, la firma per sé no implica la implementación de sus disposiciones.

Una tercera dimensión por destacar es el carácter movilizador de una vinculación estrecha con los Estados Unidos en los países de la región. En algunos casos, esto generó vínculos de tipo transnacional entre las sociedades civiles de América Latina en rechazo al acuerdo ${ }^{30}$. Sin embargo, el análisis de experiencias nacionales muestra la presencia de elementos contradictorios, con el caso de movimientos sindicales a favor de un acuerdo, de gremios empresariales en contra o, incluso, alianzas que involucran a empresarios y trabajadores en su rechazo ${ }^{31}$.

28. GONZÁLEZ (2009).

29. ALLE y ELSIG (2016); ANDERSON et al. (2011); DÜR et al. (2014); HENRIK et al. (2009); LIM et al. (2012); MANSFIELD y MILNER (2012); RODRIK (2000).

30. VON BÜLOW (2009); DOBRUSIN (2015).

31. BULL (2008); MURILLO (2001); GARCÉ y YAFFÉ (2014). 
En este sentido, la entrada en vigor de un acuerdo con EE.UU. ha activado mecanismos de participación de la sociedad civil, como por ejemplo el referéndum popular en Costa Rica, donde el CAFTA fue aprobado por un estrecho margen de un 51,6\%, contradiciendo los pronósticos que preveían su rechazo ${ }^{32}$. Esta victoria ha sido explicada por diversas razones como, por ejemplo, la movilización de las élites a votar en desmedro de otros grupos sociales ${ }^{33}$.

En consecuencia, los antecedentes expuestos denotan la relevancia de la pregunta de investigación: para el periodo 1990-2015 ¿qué variables explican que un país latinoamericano haya puesto en vigor un acuerdo comercial con Estados Unidos?

Bajo esta lógica, es importante explicar la relevancia a la hora de definir el periodo analizado. Estudiar los condicionantes de la entrada en vigor de un acuerdo comercial con Estados Unidos previo a 1990, no tendría demasiado sentido práctico, debido a que el desarrollo de la política comercial estadounidense hacia la región por medio de acuerdos comerciales es un fenómeno que emerge a partir de los años 9o's ${ }^{34}$. Asimismo, este proceso coincide con la confluencia de la democracia, la vigorización de las cadenas de valor global y las estrategias de libre mercado que se enraizaron a finales del siglo $\mathrm{XX}^{35}$.

Al mismo tiempo, es imperativo precisar que el foco de este trabajo está puesto en el estudio de los casos latinoamericanos. En este sentido, el análisis no busca explicar las características de la contraparte estadounidense, dimensión que queda pendiente para futuras investigaciones. A partir de los argumentos presentados anteriormente, vale la pena preguntarse qué llevó a que un país de América Latina suscribiera un acuerdo comercial con los Estados Unidos, en especial, tomando en cuenta las implicancias que éste conlleva.

La revisión de los antecedentes nos conduce a considerar que una mayor importancia de las reformas de mercado implementadas por los países de la región, la cercanía geográfica y comercial con Estados Unidos y la exposición de los países de América Latina al comercio internacional tendrían un efecto positivo en la entrada en vigor de un ALC con EE.UU. A partir de ello, la hipótesis del artículo es que la profundidad de las reformas de mercado que implementaron los países fue incrementando la posibilidad que un país adopte un acuerdo con los Estados Unidos, por sobre otras variables indicadas en la literatura, como la cercanía geográfica, integración comercial y exposición al comercio internacional ${ }^{36}$.

32. RODRÍGUEZ et al. (2008).

33. URBATSCH (2013).

34. WTO (2011).

35. STREECK (2012); PRZEWORSKI (1991); HAGGARD y KAUFMAN (1997).

36. RAVENHILL (2011); WTO (2011); ALLE y ELSIG (2016). 


\section{i) Definición y operacionalización de variables}

Una vez establecidos el problema de investigación, la pregunta y las hipótesis tentativas, se torna relevante enunciar las variables consideradas en este trabajo. La variable dependiente se definió como el tiempo transcurrido desde 1990 hasta que entró en vigor un acuerdo comercial entre un país de América Latina y Estados Unidos, cuya ocurrencia es codificada con un valor $=1$, mientras que cuando no entra en vigor es representada con un valor $=0$. En cuanto a las variables independientes, las ordenamos en tres grupos con propósito de evaluar las hipótesis postuladas en el apartado anterior.

Un primer grupo de variables se definió a partir del grado de profundidad de las reformas de mercado por parte de los países latinoamericanos. Para ello se especificaron dos indicadores: la adhesión a una economía de mercado y el rol del Estado en la economía.

La mayor adhesión a una economía de mercado se ha observado por medio de los resultados agregados del Índice de Libertad Económica. Estos se presentan en una escala de 1 a 100, los que se clasifican de la siguiente manera: "libre" (100-80); "mayoritariamente libre" (79,9-70); "moderadamente libre" (69.9-60); "mayoritariamente no libre" (59.9-50); "represivo" (49.9-0)37. Respecto al papel del Estado en la economía, se observa por medio de la variable State Ownership of the Economy, información obtenida del proyecto Varieties of Democracy (V-DEM). Esta se interpreta de la siguiente manera: Valores en torno a cero (o) indican una presencia casi absoluta del Estado, mientras los valores cercanos a cuatro (4) muestran una mayor importancia del sector privado ${ }^{38}$. En ambos casos, tanto una mayor adhesión a un modelo de libre mercado como una orientación pro-sector privado implicarían un mayor riesgo de entrada en un acuerdo con los EE.UU.

Otro grupo de variables que se consideraron son aquellas relativas a la geopolítica del comercio. La pertenencia de un país latinoamericano a una determinada zona de influencia de EE.UU. explicaría positivamente la entrada en vigor de un acuerdo con dicha potencia. Reformulando el criterio planteado por Raymond Aron para contextos de guerra ${ }^{39}$, la importancia geopolítica de los países latinoamericanos para los EE.UU. incrementaría el riesgo de un acuerdo comercial con la principal potencia del mundo. La definición de zonas de influencia para los Estados Unidos depende, en gran medida, de factores políticos, económicos y de coyuntura internacional, entre

37. Index of Economic Freedom, http://www.heritage.org/index/.

38. COPPEDGE et al. (2016).

39. ARON (1967). 
otros $^{40}$. Aún así, a lo largo de la historia han existido ciertas constantes en el interés de Estados Unidos por la región.

Definir zonas de influencia es relativamente difícil de precisar dado que no se estipula en un indicador estricto y su conmensurabilidad es compleja. Es por ello que definimos dos indicadores que nos permiten evaluar este argumento: la cercanía geográfica y relación comercial con Estados Unidos.

En este sentido, se tiende a pensar que la cercanía geográfica con EE.UU. disminuye los costos de transporte, facilita el comercio y, por ende, existiría un mayor interés en la entrada en vigor de un acuerdo. Siendo así, este indicador se operacionalizó a partir de la pertenencia a determinada subregión, clasificando a América Central, el Caribe y México como = o y América del Sur =1.

La dimensión geopolítica se complementa con argumentos que sitúan a la relación comercial como un factor que incide en la puesta en vigor de un ALC con la principal potencia del mundo ${ }^{41}$. Esta variable se definió a partir del ratio entre las importaciones provenientes de Estados Unidos respecto del total de ese flujo para cada país en un año dado ${ }^{42}$. Si bien, como ya se ha mencionado, este trabajo se enfoca en el estudio desde la perspectiva latinoamericana, se tendería a pensar que cuando EE.UU. tiene una mayor participación en el volumen importado de un país latinoamericano, cuenta con un incentivo para reducir costos, entre ellos aranceles y barreras no arancelarias y, por lo tanto, existiría un mayor estímulo para la entrada en vigor de un ALC.

Finalmente, el tercer grupo de variables utilizadas en este estudio se refieren al nivel de exposición al comercio internacional. Bajo esta lógica, se asume que a mayor inserción en las dinámicas comerciales globales existiría un mayor riesgo de experimentar un acuerdo con Estados Unidos. Ello se indagó a partir de dos variables: la importancia del comercio, operacionalizada como el volumen de comercio total en función del producto interno bruto ${ }^{43}$, y el número de compromisos comerciales que un país notificó ante la Organización Mundial de Comercio. A efectos de este trabajo, cuando se refiere al concepto Acuerdo Comercial o ALC se incluye en el tratamiento todos los compromisos comerciales del país estudiado. En este sentido se incorpora en dicha variable acuerdos de primera, segunda y tercera generación sin realizar diferenciación alguna entre ellos. Entre los compromisos contemplados se consideran

40. MORANDÉ (2005).

41. RAVENHILL (2011).

42. Los datos son expresados en porcentaje y fueron tomados del Banco Mundial, de la base World Integrated Trade Solutions o WITS, https://wits.worldbank.org/WITS/WITS/Restricted/Login. aspx, último acceso 20 de agosto de 2017.

43. Esto se expresa con la fórmula exportaciones/PIB. Los datos fueron tomados de la base World Development Indicators, del Banco Mundial, https://data.worldbank.org/products/wdi, último acceso 20 de agosto de 2017. 
Acuerdos de Complementación Económica, Uniones Aduaneras, Acuerdos Preferenciales de Comercio y todos aquellos instrumentos que versa en materia comercial ${ }^{44}$.

En el anexo 2 se sintetizan las características de las variables consideradas para esta investigación y su potencial efecto en el incremento del riesgo que tomaron los países latinoamericanos al experimentar un acuerdo de libre comercio con Estados Unidos.

En cuanto a la presencia de valores perdidos, el caso más problemático se encuentra en la variable Índice de Libertad Económica, pues presenta datos desde 1995, llegando a un 19\% de ausencia de información. Si el modelo se estimara sólo con aquellos casos que presenten la totalidad de datos (complete case analysis) esto reduciría considerablemente el número de observaciones ${ }^{45}$. Por ello, en este caso, se evaluó realizar una imputación a partir del valor predicho obtenido mediante el resultado de regresiones ${ }^{46}$. Dado que esta técnica no mostró una distorsión relevante respecto de la media y desviación estándar originales (anexos 3 y 4), se optó por utilizar la estimación asumiendo la presencia de los valores perdidos ${ }^{47}$.

\section{ii) Enfoque y técnica}

Para responder a estas hipótesis, se optó por un enfoque de EHA. En el campo de las relaciones internacionales, ha sido utilizado en diversas temáticas, tales como, el estudio de la sobrevivencia de un liderazgo político en el caso de conflicto bélico ${ }^{48}$, las alianzas con otros países en función a su nivel de democracia ${ }^{49} \mathrm{o}$ la ratificación de acuerdos en materia medioambiental ${ }^{50}$. También ha sido empleado en política comparada para analizar la rotación ministerial ${ }^{51} \mathrm{y}$ el término anticipado de una presidencia ante situación de crisis ${ }^{52}$.

44. Los datos fueron tomados de la base Regional Trade Agreements Informations - System, de la Organización Mundial de Comercio, https://rtais.wto.org/UI/PublicMaintainRTAHome.aspx, último acceso 22 de agosto de 2017.

45. ALLISON (2002).

46. Se utilizaron como variables auxiliares el Índice de Libertad Económica del Instituto Fraser y el Índice de Democracia (Polity IV), que presentan una correlación de Pearson de 0.76 a un 99\% de confianza con el Índice de Libertad Económica de la Fundación Heritage.

47. ALLISON (2002).

48. DE MESQUITA y SIVERSON (1995).

49. GAUBATZ (1996).

50. BERNAUER et al. (2013).

51. CAMERLO y PÉREZ - LIÑÁN (2015); GONZÁLEZ-BUSTAMANTE y OLIVARES (2016); SOMER-TOPCU Y WILLIAMS (2008).

52. MARTÍNEZ (2015). 
En materia de relaciones económicas internacionales, esta técnica ha sido implementada, mayormente, en la identificación de determinantes durante el proceso de aprobación legislativa de $\mathrm{ALC}^{53}$. Sin embargo, su aplicación es escasa en tópicos de economía política internacional como la entrada en vigor de acuerdos comerciales.

Esta característica hace del enfoque metodológico utilizado una contribución analítica, diferenciándose de otras aproximaciones, las cuales explican la probabilidad de ocurrencia del fenómeno estudiado, como modelos logit o probit ${ }^{54}$, que en este caso harían bastante problemático abordar este problema. En primer lugar, es la presencia de un evento de registro único por cada país, como es la entrada en vigor de un tratado internacional, lo que reduce el potencial de eventos (o valores $=1$ ) a los 20 países analizados. En segundo lugar, que el proceso a analizar no es la presencia del acuerdo en vigor, sino que analizar el proceso que llevó a que éste ocurriera, que en realidad remite a un evento que es dependiente de un tiempo de exposición previo. Ello hace que la aplicación de modelos para variables discretas del tipo logit o probit no sea la más apropiada, sino que más bien modelos de sobrevivencia, como los utilizados aquí ${ }^{55}$. Aplicar esta técnica al estudio de la entrada en vigor de ALC con EE.UU. nos permite identificar aquellos factores que explicarían las condiciones previas a la ocurrencia del evento.

El EHA busca explicar causas a fenómenos en un periodo temporal determinado, análisis que es conocido mayormente como análisis de sobrevivencia en la bioestadística y tiempo de falla (failure time) en el campo de la ingeniería ${ }^{56}$. La particularidad de este enfoque es que dichos factores se ubican en un momento previo a la ocurrencia del evento estudiado ${ }^{57}$.

Un evento se define como un cambio cualitativo que ocurre en un punto de tiempo. Los eventos pueden ser de registro único o múltiple durante el período de observación $^{58}$. En el primer caso es un suceso que ocurre solo una vez en el periodo de tiempo estudiado. Un ejemplo de ello es el deceso de una persona, dado que un individuo no puede experimentar la muerte en más de una oportunidad. Por otra parte, se entiende por eventos múltiples a aquellos que se reiteran en el tiempo, como sería la situación laboral de una persona, la cual puede cambiar de empleo, ser despedido o jubilar ${ }^{59}$.

53. BOX-STEFFENSMEIER et al. (1997).

54. ALLE y ELSIG (2016).

55. MILLS (2011); CLEVES et al. (2016).

56. ALLISON (2014).

57. BOX-STEFFENSMEIR et al. (2003).

58. ALLISON (2014); MILLS (2011).

59. ALLISON (2014); MILLS (2011); CLEVES et al. (2016). 
Para efectos de esta investigación la variable dependiente es el evento de registro único "entrada en vigor de un acuerdo comercial con Estados Unidos". Esto se operacionalizó mediante una variable dummy donde la entrada en vigor de un ALC con EE.UU. es $=1$, pues los países una vez que ratifican el compromiso no pueden experimentar el evento nuevamente y, por ende, son excluidos del estudio. Esta es una de las principales diferencias con otras técnicas comúnmente utilizadas para el tratamiento de variables dicotómicas en las que se estudia la probabilidad de ocurrencia de la variable dependiente a lo largo del tiempo, como por ejemplo en los modelos logit o probit ${ }^{60}$.

Una vez definido el evento, se precisa la tasa de riesgo (o hazard ratio) que, a efectos de este artículo se plantea como el ratio entre la probabilidad de ocurrencia de la entrada en vigor de un acuerdo comercial con Estados Unidos y la sobrevivencia, entendida como aquellos países latinoamericanos, que entre 1990 y 2015, no experimentaron la entrada en vigor de dichos compromisos.

Una vez definido el evento, que es la entrada en vigor de un acuerdo con los Estados Unidos, se estima una función de sobrevivencia o $S(t)$, que consiste en la proporción de sujetos u observaciones que sobreviven (no ponen en vigor un acuerdo con EE.UU. en este caso) más allá de un tiempo $t$, la que se expresa en $S(t)=\operatorname{Pr}(T \geq t)$. Esta se contrasta respecto a quienes no sobreviven (f), presentando el ratio (h) entre las unidades experimentan el evento (f) versus quienes no $(S)$, es decir $h(t)=\frac{f(t)}{S(t)}{ }_{11}$.

En ciencias sociales, el método más utilizado es la regresión de $\mathrm{Cox}^{62}$, el cual sigue un enfoque semi-paramétrico de acuerdo con Allison ${ }^{63}$. Su componente no paramétrico se define a partir de la no especificación de la función de riesgo respecto a la distribución del tiempo asociado a un evento. Al mismo tiempo, implica características paramétricas pues se implementa mediante un modelo de regresión estimado a través de una estrategia de máxima verosimilitud aunque sin estimación de una constante. Su fórmula es: $\log h(t)=\alpha(t)+\beta_{1} X_{1}+\beta_{2} X_{2}^{64}$.

Su principal desventaja es la dificultad de realizar predicciones más allá del tiempo estudiado a causa de que esta técnica no contempla la presentación de una constante en su resultado. Aun así, presenta virtudes como una menor restricción en la especificación de la función de riesgo y la estimación de parámetros consistentes ${ }^{65}$. Asimismo, el foco del artículo no está puesto en predecir el punto de tiempo en que pueda

60. ALLISON (2014); CLEVES et al. (2016).

61. MILLS (2011); ALLISON (2014).

62. COX (1972).

63. ALLISON (2014).

64. MILLS (2011); ALLISON (2014).

65. MILLS (2011); ALLISON (2014); CLEVES et al. (2016). 
ocurrir la entrada en vigor de un acuerdo, dado que es un evento inusual y de baja ocurrencia (a diferencia de cambios ministeriales, en los que si puede tener sentido), sino en que identificar variables institucionales que hacen más probable su aparición como evento. Ello nos hace optar por modelos de Cox y no por estimaciones paramétricas ${ }^{66}$.

En el caso del presente artículo, la base de datos utilizada contiene un total de 520 observaciones con estructura de panel (país-año), en los que 11 países experimentaron el evento, es decir, la entrada en vigor de un acuerdo comercial con EE.UU. durante el período de observación.

La definición del número de co-variables toma una relevancia crucial en el diseño metodológico de los modelos de sobrevivencia. Si se siguiera el criterio estricto de eventos por variable (EPV) ${ }^{67}$, nos limitaría a la construcción de un modelo que considere solo una variable explicativa. Siguiendo otros trabajos, optamos por flexibilizar esta regla lo que nos permitió agregar hasta tres (3) por cada modelo realizado ${ }^{68}$.

Para nuestra investigación, se estipularon las siguientes etapas: en primer lugar, realizamos un análisis descriptivo mediante estimador Kaplan-Meier, en el que presentamos la función de sobrevivencia. A continuación, se efectuaron una serie de regresiones bivariadas a fin de identificar y estimar la pertinencia de la inclusión de las variables explicativas previamente definidas. Finalmente, se realizó el análisis por medio de tres (3) modelos multivariados, cada uno con variables que responderían a la hipótesis planteada. A posteriori se elaboró un cuarto modelo considerando los resultados obtenidos anteriormente. El propósito de este último es dar cuenta de una mejor estimación respecto a cuáles serían aquellos factores que explican la puesta en vigor de un acuerdo comercial con los EE.UU. en el período estudiado.

Para la interpretación de los resultados en este tipo de aproximaciones, es más práctico considerar el hazard ratio (HR) dado que expresa la variación porcentual del riesgo. Por su parte el $\beta$ nos determina la direccionalidad de dicho efecto.

Para controlar problemas de heterogeneidad entre los países, utilizamos incremento de error estándar en clúster ${ }^{69}$, testeando, posteriormente, los resultados en el caso de quebrantar el supuesto de proporcionalidad en el riesgo mediante la aplicación del test de Schoenfeld.

66. CLEVES et al. (2016).

67. El criterio EPV estipula la definición de una variable independiente por cada diez eventos (1:10) GONZÁLEZ-BUSTAMANTE y OLIVARES (2016).

68. MARTÍNEZ (2015).

69. ALLISON (2009). 


\section{Resultados}

El análisis descriptivo muestra que la sobrevivencia comienza a disminuir de manera sustantiva a partir del año 17 (2006), cuando un 65\% de las unidades continúan sin un compromiso comercial con EE.UU. Para el año 33 (2012) la sobrevivencia es de $45 \%$ lo cual se mantiene hasta el año 26 (2015) (Tabla 3).

Tabla 3: Función de Sobrevivencia, países de América Latina, 1990 - 2015.

\begin{tabular}{|c|c|c|c|c|}
\hline Tiempo & \multirow{2}{*}{$\begin{array}{c}\text { Experimentan } \\
\text { evento }\end{array}$} & $\begin{array}{c}\text { Función de Sobre- } \\
\text { vivencia }\end{array}$ & \multicolumn{2}{|c|}{ Intervalo de confianza (95\%) } \\
\cline { 4 - 5 } & 1 & 0.95 & Inferior & Superior \\
\hline 5 & 1 & 0.90 & 0.69 & 0.99 \\
\hline 15 & 5 & 0.65 & 0.66 & 0.97 \\
\hline 17 & 2 & 0.55 & 0.40 & 0.81 \\
\hline 20 & 2 & 0.45 & 0.31 & 0.73 \\
\hline 23 & 0 & 0.45 & 0.23 & 0.65 \\
\hline 26 & & & 0.23 & 0.64 \\
\hline
\end{tabular}

Fuente: Elaboración propia.

Esto se complementa con el análisis descriptivo del incremento del riesgo (Figura 1), obtenido mediante estimador Nelson-Aalen. Estos resultados, no obstante, deben interpretarse con cuidado al tomar en cuenta que sus intervalos de confianza (CI) son bastante amplios.

Figura 1. Incremento en el riesgo de un acuerdo con Estados Unidos

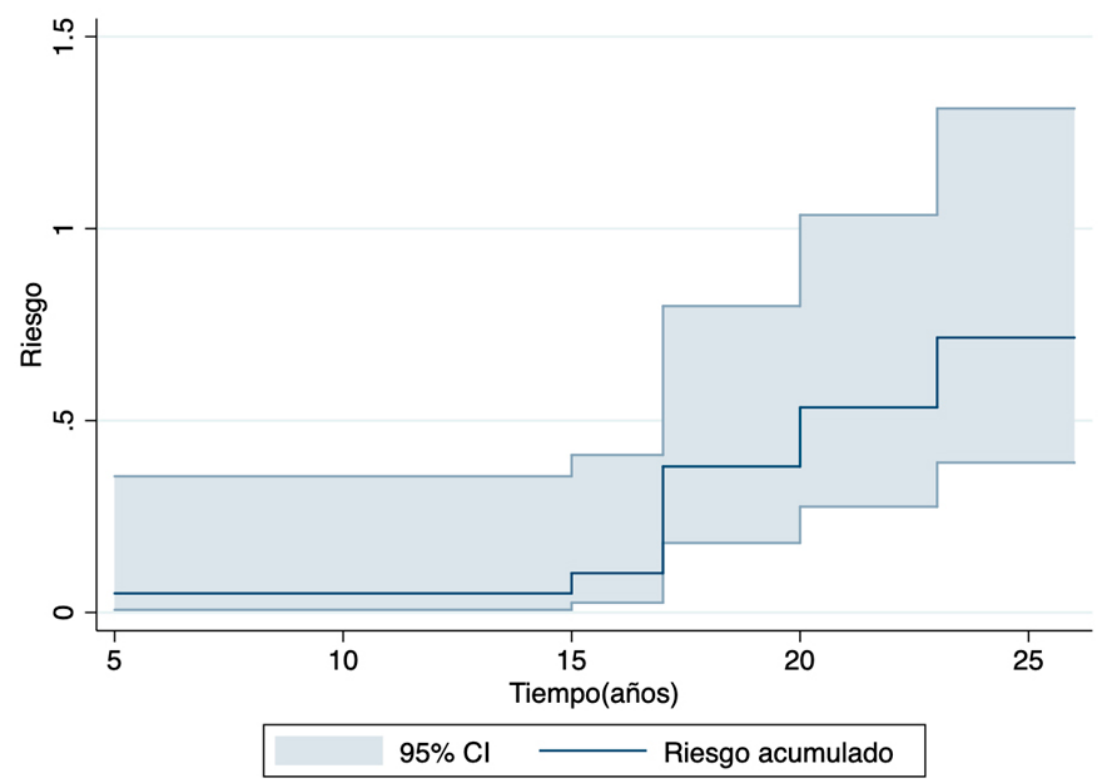

Fuente: Elaboración propia. 
Los resultados muestran que un punto de incremento en la adhesión a un modelo de libertad económica, aumenta en un $13 \%$ el riesgo (hazard ratio o $\mathrm{HR}=1.12$ ) de la entrada en vigor de un acuerdo comercial con los Estados Unidos (anexo 4). En términos de facilitar su interpretación, un valor superior a uno indica un incremento porcentual del riesgo, mientras que inferior a 1 representa una disminución.

Cabe señalar que el término riesgo no es una valoración negativa del evento, sino que responde a un término propio de la técnica, que, para efectos de este artículo es el incremento en la posibilidad que llegue a ocurrir la entrada en vigor de un acuerdo con la principal potencia del mundo. En ese sentido, se usan los términos riesgo y posibilidad de manera intercalada como sinónimos.

El aumento de un punto en la variable relevancia del sector privado en la economía incrementó el riesgo de la entrada en vigor de un acuerdo con los Estados Unidos casi nueve veces $(\mathrm{HR}=9.17)$, aunque esa lectura en realidad está indicando el paso de la categoría o a 1. De acuerdo con la región de pertenencia, los países de América del Sur disminuyeron el riesgo de la entrada en vigor de un ALC con EE.UU. en un 74\% (1-0.26) respecto a los de América Central, México y los principales estados del Caribe (anexo 4).

Asimismo, una mayor importancia de las importaciones estadounidenses aumenta el riesgo de un acuerdo comercial en un 7\%. Cada compromiso en materia de comercio que firma un país latinoamericano incrementa un $76 \%$ el riesgo de la entrada en vigor de un ALC con EE.UU. De acuerdo, a las medidas de entropía y de ajuste, esta última variable sería la más importante, dado un mayor valor del pseudo-logaritmo de máxima verosimilitud o $\mathrm{Ll}(\mathrm{M})^{70}$ y uno menor en las medidas de entropía, que se expresa en Akaike Information Criteria (AIC) y Bayesian Information Criteria (BIC) y que se usa para comparar modelos (anexo 4). En términos de interpretación, un menor valor, significa un mejor ajuste del modelo, que equivale a señalar, una mayor reducción de los niveles de entropía ${ }^{71}$.

La apertura del comercio tiene un impacto bajo en el incremento en el riesgo de experimentar un acuerdo con los Estados Unidos, de un $1 \%$ por cada punto que aumenta la exposición de los países al comercio internacional, con un 77\% de confianza. Si bien este resultado no haría aconsejable su inclusión en el análisis multivariado, se optó por incorporar esta variable mediante una interacción con la variable región de pertenencia. Se entiende como interacción a la inclusión de una variable $Z$ que media en el efecto de una variable independiente $X$ sobre una dependiente $Y^{72}$.

70. Respecto del modelo nulo, aquel que no incluye variables independientes.

71. CLEVES et al. (2016).

72. JACCARD y TURRISI (2003). 
El análisis multivariado se realizó comparando tres modelos, a partir de la selección realizada en un análisis bivariado. En el primero, se evaluó el impacto de las reformas de mercado, el segundo la cercanía comercial y geográfica con EE.UU. y el tercero la inserción comercial de los países latinoamericanos.

De acuerdo con las pruebas de ajuste, el modelo que mejor explicó el fenómeno estudiado es el M4 (Tabla 4), donde la inclusión de variables, según el Ll(M), presenta un mayor ajuste de los tres respecto de un modelo nulo. En todos los casos anteriores se aplicó el test de residuos de Schoenfeld, que confirmó el supuesto de proporcionalidad en la distribución de los riesgos ${ }^{73}$.

Una mayor orientación hacia la libertad económica aumentó el riesgo de la entrada en vigor un acuerdo con Estados Unidos, dado que cada punto del IEF incrementó el riesgo (HR) en un $12 \%$, como se ve en modelo 1 . Si bien este no tiene un nivel de confianza del 95\% sí lo sería a un 90\% ( $p<0.08)$. Por cada aumento en un punto de la relevancia del sector privado, la posibilidad de un acuerdo de libre comercio aumenta 10 veces (Tabla 4 ).

En cuanto a las variables geopolíticas (M2), los países de América del Sur (AS) presentaron un 53\% menos de riesgo que los de América Central, México y las principales islas del Caribe en experimentar la puesta en vigor de un acuerdo con EE.UU. aunque presentó apenas un $75 \%$ de confianza. Cada punto porcentual de aumento de la importancia de las exportaciones estadounidenses representó un 5\% de incremento en que llegue a ocurrir un tratado comercial (Tabla 4).

Respecto de las variables de inserción comercial (M3), cada acuerdo en vigor incrementó en un 89\% el riesgo de un acuerdo. En el caso de una mayor exposición al comercio internacional, al estar mediada por la región de pertenencia, para el caso de los países de América del Sur, cada punto de mayor exposición al comercio internacional redujo la posibilidad de un tratado con EE.UU. en un $2 \%$, mientras que para el caso de los de América Central, México y el Caribe, éste se redujo en un $2 \%$. Si bien su nivel de confianza es de un $86 \%$, aumentó respecto de los resultados del análisis bivariado en nueve puntos (Tabla 4).

Finalmente, en vista de los resultados obtenidos, planteamos un cuarto modelo, en el cual incorporamos las variables relevantes de cada uno de los anteriores: del M1 se tomó la variable Índice de Libertad Económica pese a su bajo $p$ valor dado que los resultados de la adhesión a un modelo de libertad económica podrían estar dependiendo de una reducción de la importancia del Estado como agente económico (Tabla 4).

73. Respondiendo a los supuestos del modelo de Cox donde la hipótesis nula $\left(\mathrm{H}_{0}\right)$ significa que los riesgos se distribuyen de manera proporcional, un abordaje desde esta técnica requiere la aceptación de la H0. A efectos de nuestro trabajo ello fue confirmado para M1, M2, M3 y M4. 
Del M2 y M3 se incorporaron las variables relevancia de las importaciones provenientes de EE.UU. y número de ALC en vigor, respectivamente, a causa de su significancia estadística (Tabla 4).

Tabla 4: Análisis multivariado.

\begin{tabular}{|c|c|c|c|c|}
\hline & $\begin{array}{l}\text { Reforma de } \\
\text { Mercado (M1) }\end{array}$ & $\begin{array}{l}\text { Área de influen- } \\
\text { cia (M2) }\end{array}$ & $\begin{array}{l}\text { Inserción com- } \\
\text { ercial (M3) }\end{array}$ & $\begin{array}{l}\text { Modelo final } \\
\text { (M4) }\end{array}$ \\
\hline & HR & $\mathrm{HR}$ & HR & HR \\
\hline IEF & $\begin{array}{ll}0.110 & \\
(0.063) & 1.116\end{array}$ & -- & -- & $\begin{array}{l}-0.182^{* * *} \\
(0.040)\end{array}$ \\
\hline $\begin{array}{l}\text { Relevancia del } \\
\text { sector privado en } \\
\text { la economía }\end{array}$ & $\begin{array}{ll}2.306^{\star *} & \\
(0.859) & 10.03\end{array}$ & -- & -- & \\
\hline $\begin{array}{l}\text { Subregión } \\
(\mathrm{AS}=1)\end{array}$ & -- & $\begin{array}{ll}-0.806 & \\
(0.693) & 0.447\end{array}$ & -- & \\
\hline $\begin{array}{l}\text { Importaciones } \\
\text { EE.UU. (\%) }\end{array}$ & -- & $\begin{array}{ll}0.047^{\star} & \\
(0.023) & 1.05\end{array}$ & -- & $\begin{array}{ll}0.087^{\star * \star} \\
(0.022) & 1.09\end{array}$ \\
\hline $\begin{array}{l}\text { Apertura de } \\
\text { comercio }\end{array}$ & -- & -- & $\begin{array}{ll}-0.016 & \\
(0.011) & 0.984 \\
\end{array}$ & \\
\hline $\begin{array}{l}\text { Número de } \\
\text { compromisos } \\
\text { comerciales }\end{array}$ & -- & -- & $\begin{array}{ll}0.641^{* * *} \\
(0.170) & 1.89\end{array}$ & $\begin{array}{ll}0.579^{* * *} \\
(0.105) & 1.78\end{array}$ \\
\hline $\begin{array}{l}\text { Apertura de } \\
\text { comercio* }^{*} \\
\text { subregión }\end{array}$ & -- & -- & $\begin{array}{ll}-0.035^{\star} & \\
(0.016) & 0.97\end{array}$ & \\
\hline $\begin{array}{l}\text { IEF* relevancia }^{*} \\
\text { del sector privado }\end{array}$ & & & & $\begin{array}{l}0.064^{* * *} \\
(0.009) \quad 1.07\end{array}$ \\
\hline $\mathrm{P}>\mathrm{Chi} 2$ & 0.001 & 0.020 & 0.001 & 0.000 \\
\hline $\mathrm{Ll}(\mathrm{M})$ & -21.42 & -25.90 & -20.98 & -15.85 \\
\hline $\mathrm{BIC}$ & 54.35 & 63.75 & 60.05 & 54.45 \\
\hline Sujetos & 19 & 20 & 20 & 20 \\
\hline Eventos & 10 & 11 & 11 & 10 \\
\hline Observaciones & 315 & 394 & 416 & 296 \\
\hline
\end{tabular}

$" p<0.05 ;$;" $p>0.01 ;$;*** $p>0.000$

Fuente: Elaboración propia 
En la Tabla 4 se presentan los resultados obtenidos en el M4. Estos confirman la información obtenida en el $\mathrm{M} 2$ en la que se expone que a mayor importancia de las exportaciones de EE.UU. aumenta el riesgo de experimentar la puesta en vigor de un acuerdo con la principal potencia del mundo en un 9\%. Lo mismo ocurre con el aumento en el número de acuerdos comerciales, donde cada compromiso lo incrementa en un $78 \%$. Finalmente, la adhesión a un modelo económico de libre mercado, cuando depende de una mayor orientación hacia el sector privado como principal agente, aumenta el riesgo en un $7 \%$ por cada punto de aumento. En términos de ajuste, éste presenta un mayor poder explicativo por parte de las variables consideradas, al incrementarse la diferencia respecto de un modelo nulo y manteniéndose los niveles de entropía, observados en la reducción del BIC respecto del M1.

En la Figura $2^{74}$ observamos la estimación del riesgo a partir de los resultados del M4, en el cual se observa un incremento del riesgo de la entrada en vigor de un ALC con EE.UU. a partir de año 17 (2006). Este alcanza su punto máximo en el año 19 (2008), para, posteriormente, disminuir el efecto que toman sobre el riesgo las covariables consideradas para el resto del periodo estudiado.

Figura 2: Riesgo Acumulado.

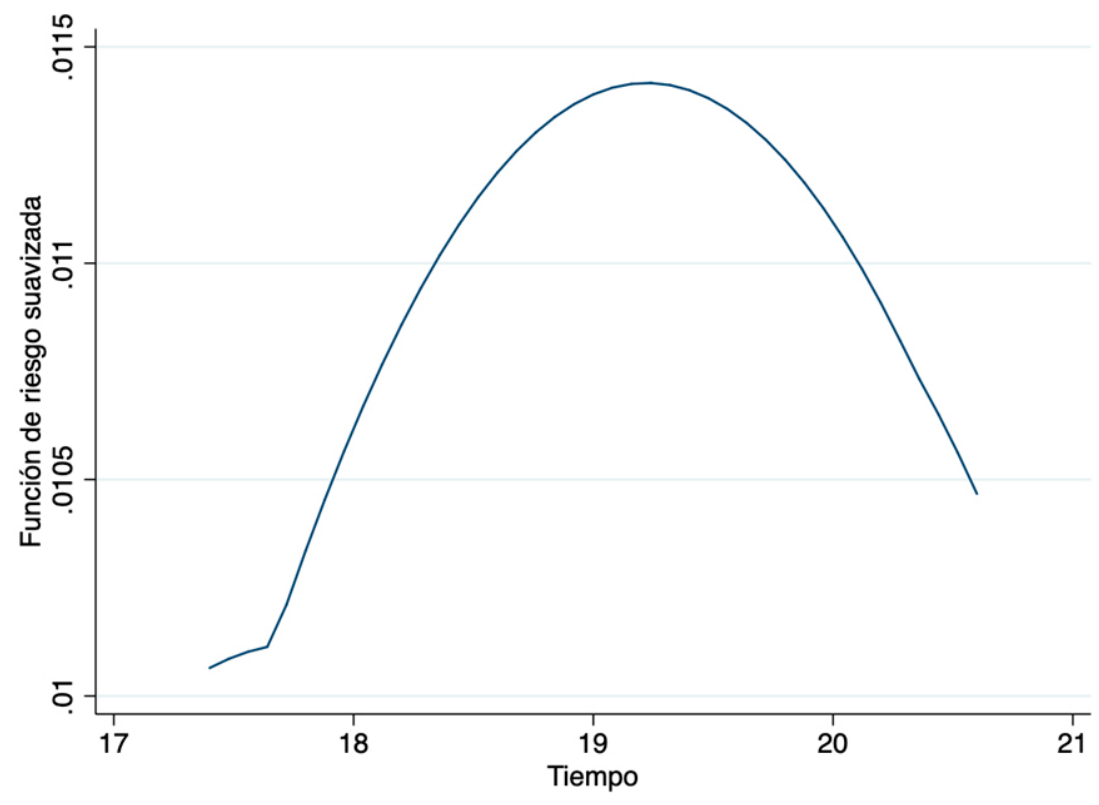

Fuente: Elaboración propia.

74. Esta Figura tiene la particularidad que muestra un efecto no lineal del incremento del riesgo de nuestra variable dependiente al incluir todas las variables independientes seleccionadas para el M4. 
En la Figura3 observamos el contraste entre las funciones de sobrevivencia obtenidas mediante la estimación de la regresión de Cox (resultado de la estimación o predicho) y de Kaplan-Meier ${ }^{75}$ (que presenta el valor observado en los datos). Los resultados del modelo muestran una subestimación de la sobrevivencia, para el caso de los países de América del Sur, situación que es a la inversa para los casos de aquellas naciones fuera del subcontinente.

Figura 3: Comparación estimación (modelo de Cox) y datos observados (KarpanMeier).

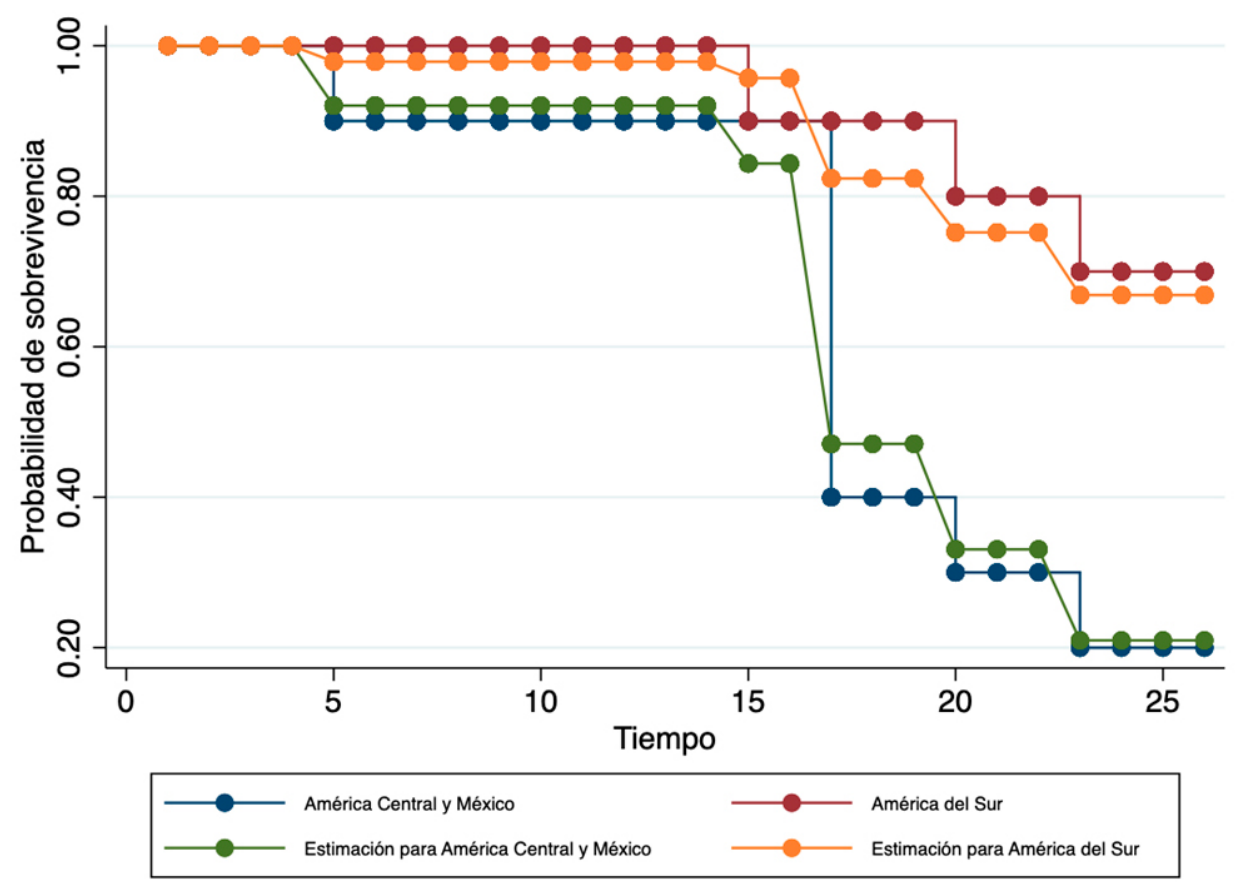

Fuente: Elaboración propia.

75. La Figura 3 tiene por objeto comparar los valores predichos con los observados en los datos. Cuanto mayor sea el ajuste entre ambos, más eficiente es el modelo propuesto. 


\section{Conclusiones}

A partir del planteamiento de nuestro problema de investigación, podemos afirmar que entre 1990 y 2015, los países de América Latina han presentado distintos énfasis en sus esquemas de integración comercial con el mundo. Una vinculación estrecha con los Estados Unidos es un elemento diferenciador en dicho proceso dadas las complejidades que este implica a la hora de negociar un acuerdo comercial y los efectos de este en materia de política interna.

Considerando los hallazgos obtenidos en la modelación de nuestro problema, una mayor tendencia hacia un modelo de libre mercado se confirma como elemento explicativo de la entrada en vigor de un acuerdo comercial con EE.UU. cuando está mediado por un proceso paralelo de transformación en que el sector privado adquiere un rol más destacado como actor económico en detrimento del Estado. El elemento mencionado anteriormente confirma los argumentos que señalan que la presencia de este tipo de acuerdos tiende a reforzar o acelerar procesos de reforma económica ${ }^{76}$. Bajo la misma lógica de Allee y Elsig77, la tesis que sostiene que los compromisos comerciales son elementos que refuerzan las estrategias de reforma de mercado, nuestros hallazgos complementan a la literatura mencionada, destacándose por enfatizar el proceso previo a la entrada en vigor de un acuerdo comercial con Estados Unidos.

Si bien factores como la pertenencia a una determinada zona geográfica de mayor cercanía a los Estados Unidos es un argumento presente en la literatura ${ }^{78}$, al estudiar el periodo previo a la entrada en vigor de un compromiso comercial con EE.UU. hay otros factores que se tornan más relevantes. En este sentido, y en función a nuestra hipótesis geopolítica, el peso de las importaciones estadounidense en los flujos de comercio exterior de un país latinoamericano explica consistentemente la entrada en vigor, cumpliéndose de manera parcial.

Junto con ello y en la misma línea de los hallazgos obtenidos por Baccini y Urpelainen $^{79}$, una mayor exposición a dinámicas de vinculación con los mercados internacionales, expresada en la variable comercio como porcentaje del PIB, no tiene un efecto estadísticamente significativo sobre la entrada en vigor de un acuerdo comercial con la principal potencia del mundo. Sin embargo, cuando definimos el nivel de inserción por medio del indicador número de acuerdos comerciales en vigor, sí se manifiesta un incremento importante en el riesgo de la entrada en vigor de un ALC con EE.UU. En este sentido, podemos inferir que una trayectoria previa en materia de vinculación

76. MANSFIELD y MILNER (2012); GATHII (2011); RAVENHILL (2011).

77. ALLE y ELSIG (2016).

78. DURAND (2000); GIRÓN (2015).

79. BACCINI y URPELAINEN (2014). 
comercial por medio de mecanismos formales es un elemento substancial para la vinculación comercial con Washington.

Asimismo, estos resultados nos permiten pensar en las estrategias de vinculación comercial de los países latinoamericanos. No obstante, la entrada en vigor de un ALC con EE.UU. podría tener relación con la estrategia de apertura comercial unilateral, argumento que no podemos confirmar estadísticamente dado que este trabajo no se ha enfocado en las disposiciones enunciadas en cada compromiso. Este punto nos lleva a considerar tanto la liberalización unilateral como las disposiciones de los acuerdos como una dimensión clave para incluir en futuras investigaciones.

\section{Referencias bibliográficas}

ALLE, Todd y ELSIG, Manfred (2016): Why Do Some International Institutions Contain Strong Dispute Settlement Provisions? New Evidence from Preferential Trade Agreements. The Review of International Organizations, año 11, № 1, pp. 89-120.

ALLISON, Paul. (2002): Missing data: Quantitative applications in the social sciences. Journal of Mathematical and Statistical Psychology, año 55, №1, pp. 193-196.

ALLISON, Paul. (2009): Fixed Effects Regression Models. (London: SAGE).

ALLISON, Paul. (2014): Event History and Survival Analysis. (Washington: SAGE).

ANDERSON, Robert. D., PELLETIER, Philippe, OSEI-LAH, Kodjo., y MÜLLER, Anna Caroline. (2011): Some New Data Sources, Provisional Estimates, and an Evaluative Framework for Individual WTO Members Considering Accession: Ginebra: WTO Staff Working Paper. WTO Staff Working Paper.

ARON, Raymond. (1967): What is a Theory of International Relations. Journal of International Relations, año 21, No2, pp. 185-206.

BACCINI, Leonardo, DÜR, Andreas y ELSIG, Manfred (2018): Intra-Industry Trade, Global Value Chains, and Preferential Tariff. International Studies Quarterly, o, $1-12$.

BACCINI, Leonardo y URPELAINEN, Johannes (2014): International institutions and domestic politics: can preferential trading agreements help leaders promote economic reform? The Journal of Politics, año 76, No 1, pp. 195-214.

BALDWIN, David (1980): Interdependence and power: a conceptual analysis. International Organization, año 34, No 4,pp. 471-506.

BECK, Nathaniel y KATZ, Jonathan N. (1995): What to Do (and not to Do) with Time Series Cross Section Data. American Political Science Review, año 89 No 3, pp. 634-647. 
BERNAUER, Thomas, BÖHMELT, Tobias y KOUBY, Vally. (2013): Is There a Democracy-Civil Society Paradox in Global Enviromental Governance? Global Environmental Politics, año 13 No1, pp. 88-107.

BLISS, Harry y RUSSET, Bruce (1998): Democratic Trading Partners: The Liberal Connection, 1962-1989. The Journal of Politics, año 6o No 4, pp. 1126-1147.

BOX-STEFFENSMEIER, Janet M, ARNOLD, Laura W y ZORN, Christopher J. W. (1997): The strategic timing of position taking in Congress: A study of the North American Free Trade Agreement. American Political Science Review año 91 No 2 , pp. 324-338.

BOX-STEFFENSMEIER, Janet M, REITER, Dan y ZORN, Christopher. (2003): Nonproportional hazard and event history analysis in international relations. Journal of Conflict Resolution, pp. 33-53.

BULL, Benedicte. (2008): Policy networks and business participation in free trade negotiations in Chile. Journal of Latin American Studies, año $40 \mathrm{~N}^{\circ} 2$, pp. 195-224.

CAMERLO, Marcelo y PERÉZ-LIÑÁN, Aníbal. (2015): The politics of minister retention in presidential systems: Technocrats, partisans, and government approval. Comparative Politics, año $47 \mathrm{~N}^{\circ} 3$, pp. 315-55.

CLEVES, Mario, GOULD, William y MARCHENKO, Yulia. (2016): An Introduction to Survival Analysis Using Stata, Revised Third Edition. (College Station, Texas: Stata Press).

COPPEDGE, Michael, GERRING, John, LINDBERG, Staffan, SKAANING, SvendErik, TEORELL, Jan, ALTMAN, David, ANDERSSON, Frida, BERNHARD, Michael, FISH, Steven, GLYNN, Adam, HICKEN, Allen, HENRIK KNUTSEN, Carl, MAC MANN, Kelly, MECHKOVA, Valeriya, MIRI, Farhard, PAXTON, Pamela, PEMSTEIN, Daniel, SIGMAN, Rachel, STATON, Jeffrey y ZIMMERMAN, Briggite (2016): Varieties of Democracy Codebook v.6. (Gotemburgo: University of Gotemburgo - University of Notre-Dame).

COX, David R. (1972): Regression models and life tables (with discussion). Journal of the Royal Statistical Society, 34.

CUEVAS, Rodrigo (2019): Reformas de Mercado y Acuerdos Comerciales en América Latina (1970-2015). Revista mexicana de ciencias políticas y sociales, año 64 № 237, pp. 377-407.

DE MESQUITA, Bruce B. y SIVERSON, Randolph M. (1995): War and the survival of political leaders: A comparative study of regime types and political accountability. American Political Science Review, año $89 \mathrm{~N}^{\circ} 4$, pp. 841-855. 
DOBRUSIN, Bruno. (2015): Transnational labor action in Latin America: from the struggle against the Free Trade Area of the Americas to the return of labor relation. Labor History, año $56 \mathrm{~N}^{\circ}$ 3, pp. 270-291.

DÜR, Andreas, BACCINI, Leonardo y ELSIG, Manfred. (2014): The Design of International Trade Agreements: Introducing a New Dataset. The Review of International Organizations, año $9 \mathrm{~N}^{\circ}$ 3, pp. 353-375.

DURAND, Jorge. (200o): Tres premisas para entender y explicar la migración México-Estados Unidos. Estudios de historia y sociedad, año XXI No 83, pp. 19-35.

FFRENCH-DAVIS, Ricardo (2005): Reformas para América Latina después del fundamentalismo neoliberal. (Santiago: CEPAL).

GARCÉ, Adolfo y YAFFÉ, Jaime. (2014): Tercer acto. La era progresista. Hacia un nuevo modelo de desarrollo (Uruguay: Editorial Fin de Siglo).

GATHII, James T. (2011): The Neoliberal Turn in Regional Trade Agreements. Washington Law Review, año $86 \mathrm{~N}^{\circ} 3$, pp. 421-474.

GARTZKE, Erik (1998): Kant We All Just Get Along? Opportunity, Willingness and the Origins of the Democratic Peace? American Journal of Political Science, año $42 \mathrm{~N}^{\circ} 1$,pp. 1-27.

GARTZKE, Erik (2007): The Capitalist Peace American. Journal of Political Science, año $5 \mathrm{~N}^{\circ} 1$, pp. 166-191.

GAUBATZ, Kurt T. (1996): Democratic states and commitment in international relations. International Organization, año 50 Noo1, pp. 109-139.

GIRÓN, Alicia. (2015): Zonas libres de comercio. ¿Un camino para el desarrollo? Revista Latinoamericana de Economía, año 46 No 180 .

GONZÁLEZ, Anabel. (2009): La implementación de acuerdos comerciales preferenciales en América Latina: la experiencia de implementación del CAFTA-RD en Costa Rica. Sector Integración y Comercio, Documento de Políticas 104: Banco Interamericano de Desarrollo.

GONZÁLEZ-BUSTAMANTE, Bastián y OLIVARES, Alejandro (2016): Cambios de gabinete y superviviencia de los ministros en Chile durante los gobiernos de la Concertación (1990-2010). Colombia Internacional, año 87, pp. 81-108.

HAGGARD, Stephan y KAUFMAN, Robert. (1997): The Political Economy of Democratic Transitions. Comparative Politics, año $29 \mathrm{~N}^{\circ} 3$, pp. 263-283.

HENRIK, Henrik, MAVROIDIS, Petros C, y SAPIR, Andre. (2009): Beyond the WTO? An Anatomy of EU and US Preferential Trade Agreements. Bruselas: Bruegel Blue Print Series. 
HÜHNE, Philippe, MEYER, Birgit, NUNNENKAMP, Peter, y ROY, Martin. (2015): Democracies Cooperate More: even where it threatens to bite? Applied Economics Letters, año 23 No11, pp. 812-815.

JACCARD, James y Turrisi, Robert (2003): Interaction effects in multiple regression. (London: Sage).

KAROL, David. (2007): Does Constituency Size Affect Elected Officials' Trade Policy Preferences? The Journal of Politics, año $69 \mathrm{~N}^{\circ} 2$, pp. 483-494.

LIM, C.L, ELMS, Deborah y LOW, Patrick (editores) (2012): Trans-Pacific Partnership: The Quest for a 21st Century Trade Agreement. (Cambridge: Cambridge University Press).

McMILLAN, Susan (1997): Interdependence and conflict. Mershon International Studies Review, año $41 \mathrm{~N}^{\circ} 1$, pp. 33-58.

MANSFIELD, Edward D y MILNER, Helen V. (2012): Votes, Vetoes, and the Political Economy of International Trade Agreements. (Princeton: Princeton University Press).

MANSFIELD, Edward D, MILNER, Helen V. y PEVEHOUSE, Jon C. (20o8): Democracy, Veto Players and the Depth of Regional Integration. World Economy, año 31 $\mathrm{No} 1$, pp. 67-96.

MARSHALL, Monty G. (2013): POLITY IV Project. Political Regime and Transitions, 1800-2013. Data User's Manual. Societal-Systems Research Inc.

MARTÍNEZ, Christopher (2015): Presidential survival in South America: Rethinking the role of Democracy. International Political Science Review, año 38 No 1 , pp. 4055.

MILLS, Melinda. (2011): Introducing survival and event history analysis. (London: Sage Publications).

MORANDÉ, José. (2005): Estados Unidos y la Unión Europea en el siglo XXI: fundamentos y alcances de una unidad y diversidad. Estudios Internacionales, año 38 No 149 .

MURILLO, María Victoria (2001): Labor unions, partisan coalitions, and market reforms in Latin America. (Cambridge University Press).

PICKUP, Mark. (2015): Introduction to Time Series Analysis. (London: SAGE).

POLACHEK, Solomon W. (1997): Why Democracies Cooperate More and Fight Less: The Relationship Between International Trade and Cooperation. Review of International Economics, año 5 No 3, pp. 295-309. 
PRZEWORSKI, Adam. (1991): Democracy and the Market: Political and Economic Reforms in Eastern Europe and Latin America. (New York: Cambridge University Press).

RAVENHILL, John. (2011): Global political economy. (New York: Oxford University Press).

RODRÍGUEZ, Florisabel, GÓMEZ-BARRANTES, Miguel, y CHACÓN, Wendy. (2008): El referéndum del TLC en Costa Rica. Revista de Ciencias Sociales (Cr), pp. 49-69.

RODRIK, Dani. (200o): Trade Policy Reform as Institutional Reform. (Washington: Banco Interamericano de Dessarrollo).

RUGGIE, John G. (1982): International Regimes, Transactions, and Change: Embedded Liberalism in the Postwar Economic Order. International Organization, año $36 \mathrm{~N}^{\circ} 2$, pp. 379-415.

SOMER-TOPCU, Zeynep y WILLIAMS, Laron. (2008): Survival of the Fitest? Cabinet Duration in Postcommunist Europe. Comparative Politics, año $40 \mathrm{~N}^{\circ} 3$, pp. 313-329.

STOKES, Susan (2001): Mandates and Democracy: Neoliberalism by Surprise in Latin America. (New York: Cambridge University Press).

STREECK, Wolfgang (2012): How to Study Contemporary Capitalism? European Journal of Sociologý, año53 Noo1, pp. 1-28.

TEICHMAN, Judith (2011): The Politics of Freeing Markets in Latin America: Chile, Argentina, and Mexico. (North Carolina: The University of North Carolina Press).

URBATSCH, Robert (2013): A Referendum on Trade Theory: Voting on Free Trade in Costa Rica. International Organization, año 67 No 01, pp. 197-214.

VON BÜLOW, Marissa. (2009): Networks of Trade Protest in the Americas: Toward a new labor internationalism? Latin American Politics and Society año $51 \mathrm{~N}^{\circ} 2$, pp. $1-28$.

WTO (2011): World Trade Report: The WTO and preferential trade agreements: From co-existence to coherence. Geneve: World Trade Organization. 


\section{Anexos}

Anexo 1: Principales características económicas y políticas de países considerados (2016).

\begin{tabular}{|l|c|c|c|c|c|c|}
\hline & $\begin{array}{c}\text { TLC } \\
\text { con } \\
\text { Estados } \\
\text { Unidos }\end{array}$ & $\begin{array}{c}\text { Apertura } \\
\text { del Comer- } \\
\text { cio (\% PIB) }\end{array}$ & Ingreso & $\begin{array}{c}\text { Nivel de } \\
\text { democracia }\end{array}$ & $\begin{array}{c}\text { Índice } \\
\text { de Libertad } \\
\text { Económica }\end{array}$ & $\begin{array}{c}\text { F u n ci on - } \\
\text { miento } \\
\text { de } \\
\text { las } \\
\text { Economías } \\
\text { de Mercado }\end{array}$ \\
\hline Argentina & No & 26,1 & Medio alto & 9 & 50,4 & 5,75 \\
\hline Bolivia & No & 56,6 & Medio bajo & 7 & 47,7 & 5,86 \\
\hline Brasil & No & 24,6 & Medio alto & 8 & 52,9 & 7,14 \\
\hline Chile & Sí & 56,1 & Alto & 10 & 76,5 & 8,39 \\
\hline Colombia & Sí & 34,7 & Medio alto & 7 & 69,7 & 6,64 \\
\hline Costa Rica & No & 63,5 & Medio alto & 10 & 65 & 7,79 \\
\hline Cuba & No & 44,3 & Medio alto & -7 & 33,9 & 4,61 \\
\hline $\begin{array}{l}\text { Rep. Domini- } \\
\text { cana }\end{array}$ & Sí & 54,1 & Medio alto & 8 & 62,9 & 5,79 \\
\hline Ecuador & No & 39,0 & Medio alto & 5 & 49,3 & 5,64 \\
\hline El Salvador & Sí & 64,2 & Medio bajo & 8 & 64,1 & 6,5 \\
\hline Guatemala & Sí & 46,8 & Medio bajo & 8 & 63 & 5,11 \\
\hline Honduras & Sí & 100,7 & Medio bajo & 7 & 58,8 & 5,32 \\
\hline Jamaica & No & 78,4 & Medio alto & 9 & 69,5 & 6,11 \\
\hline México & Sí & 78,1 & Medio alto & 8 & 63,6 & 6,5 \\
\hline Nicaragua & Sí & 95,9 & Medio bajo & 9 & 59,2 & 5,46 \\
\hline Panamá & Sí & 94,3 & Medio alto & 9 & 66,3 & 7,04 \\
\hline Paraguay & No & 81,5 & Medio alto & 9 & 62,4 & 6,0 \\
\hline Perú & Sí & 44,8 & Medio alto & 9 & 68,9 & 7,18 \\
\hline Uruguay & No & 41,5 & Alto & 10 & 69,7 & 8,57 \\
\hline Venezuela & No & 54,3 & Medio alto & 4 & 27 & 4,07 \\
\hline Fuente: Ela & & & & & \\
\hline
\end{tabular}

Fuente: Elaboración propia con datos del Banco Mundial, Polity IV, Fundación Heritage y

Fundación Bertlesmann. 
Anexo 2: Variables independientes consideradas.

\begin{tabular}{|c|c|c|c|}
\hline Variable & Descripción & Operacionalización & Efecto esperado \\
\hline 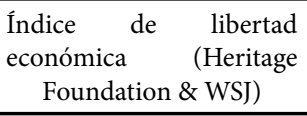 & $\begin{array}{l}\text { Grado de adhesión a } \\
\text { principios de libertad } \\
\text { económica }\end{array}$ & Valores entre 0 y 100 (\%) & $\begin{array}{c}\text { Mayor adhesión a libre } \\
\text { mercado, mayor riesgo } \\
(+,+)\end{array}$ \\
\hline $\begin{array}{l}\text { Relevancia del sector pri- } \\
\text { vado en la economía (V- } \\
\text { DEM) }\end{array}$ & $\begin{array}{l}\text { Mide la participación del } \\
\text { estado en la economía } \\
\text { mediante la posesión de } \\
\text { activos }\end{array}$ & $\begin{array}{l}\text { Estimación de inferencia } \\
\text { bayesiana a partir de una } \\
\text { escala ordinal, donde } 0 \\
\text { es un papel monopólico } \\
\text { del Estado mientras que } 4 \\
\text { muestra un papel prepon- } \\
\text { derante del sector privado }\end{array}$ & $\begin{array}{l}\text { Mayor índice, mayor ries- } \\
\text { go, }(+,+)\end{array}$ \\
\hline Sub-Región & $\begin{array}{c}\text { Pertenencia de los países a } \\
\text { subregión }\end{array}$ & $\begin{array}{l}\text { Variable dummy }(1= \\
\text { América del Sur; } 0=\text { Amé- } \\
\text { rica Central, el Caribe y } \\
\text { México) }\end{array}$ & $\begin{array}{l}\text { Mayor cercanía geográ- } \\
\text { fica, mayor riesgo }(+,+)\end{array}$ \\
\hline $\begin{array}{l}\text { Relevancia de las Import- } \\
\text { aciones de USA (WITS) }\end{array}$ & $\begin{array}{l}\text { Participación de las im- } \\
\text { portaciones de EE.UU. re- } \\
\text { specto al total importado. }\end{array}$ & Valores entre 0 y 100 (\%) & $\begin{array}{c}\text { Mayor relevancia im- } \\
\text { portaciones, mayor riesgo } \\
(+,+)\end{array}$ \\
\hline $\begin{array}{c}\text { Apertura de Comercio } \\
\text { (WDI) }\end{array}$ & $\begin{array}{l}\text { Importancia del comercio } \\
\text { en relación al PIB }\end{array}$ & $\begin{array}{c}(\mathrm{X}+\mathrm{M}) / \mathrm{PIB} \text {. Valores entre } \\
0 \text { y } 100(\%)\end{array}$ & $\begin{array}{c}\text { Mayor exposición al com- } \\
\text { ercio internacional, mayor } \\
\text { riesgo }(+,+)\end{array}$ \\
\hline $\begin{array}{l}\text { Número de acuerdos com- } \\
\text { erciales en vigor (OMC) }\end{array}$ & $\begin{array}{l}\text { Número de compromisos } \\
\text { comerciales en un año } t_{i}\end{array}$ & Variable conteo. & $\begin{array}{l}\text { Mayor número de acuer- } \\
\text { dos, mayor riesgo }(+,+)\end{array}$ \\
\hline
\end{tabular}

Fuente: Elaboración propia en función a los indicadores mencionados en el texto.

Anexo 3: Características de variables independientes.

\begin{tabular}{|l|c|c|c|c|}
\hline & Media & Desviación & \multicolumn{2}{|c|}{ Valores perdidos } \\
\cline { 4 - 5 } & & estándar & $\mathbf{N}$ & $\%$ \\
\hline Índice de libertad económica (IEF) & 60.1 & 10.3 & 100 & 19.2 \\
\hline $\begin{array}{l}\text { Índice de libertad económica (IEF) (Im- } \\
\text { putada) }\end{array}$ & 59.6 & 9.7 & 0 & 0 \\
\hline Relevancia del sector privado en la economía & 2.91 & 0.7 & 26 & 5 \\
\hline Subregión (América del Sur = 1) & 0.5 & 0.5 & 0 & 0 \\
\hline Relevancia de las importaciones de EE.UU. & 27.7 & 15.0 & 34 & 6.5 \\
\hline Apertura de Comercio & 64.2 & 32.7 & 12 & 2.3 \\
\hline Número de acuerdos comerciales en vigor & 4.1 & 3.7 & 0 & 0 \\
\hline
\end{tabular}

Fuente: Elaboración propia. 
Anexo 4: Análisis exploratorio de variables independientes.

\begin{tabular}{|c|c|c|c|c|c|c|c|c|}
\hline & HR & $\boldsymbol{\beta}$ & Ll (M) & AIC & BIC & Sub & Fail & Obs \\
\hline IEF & $1.13^{\star}$ & $\begin{array}{c}0.126^{\star} \\
(.052)\end{array}$ & -23.86 & 49.73 & 53.51 & 19 & 10 & 325 \\
\hline IEF (Imputada) & $1.12^{\star}$ & $\begin{array}{c}0.11^{*} \\
(0.049)\end{array}$ & -27.33 & 56.66 & 60.71 & 20 & 11 & 425 \\
\hline $\begin{array}{c}\text { Mayor participación del sec- } \\
\text { tor privado en la economía }\end{array}$ & $9.17^{* *}$ & $\begin{array}{c}2.22^{* *} \\
(0.821)\end{array}$ & -26.58 & 58.22 & 62.28 & 20 & 11 & 425 \\
\hline $\begin{array}{c}\text { Subregión } \\
\text { (AS = 1) }\end{array}$ & $0.26^{*}$ & $\begin{array}{c}-1.34^{*} \\
(0.635)\end{array}$ & -28.11 & 55.16 & 59.19 & 20 & 11 & 425 \\
\hline $\begin{array}{c}\text { Importaciones EE.UU. (\%) } \\
\text { Apertura de Comercio }\end{array}$ & $1.07^{* *}$ & $\begin{array}{c}0.064^{* *} \\
(0.022)\end{array}$ & -26.33 & 54.65 & 58.63 & 20 & 11 & 394 \\
\hline $\begin{array}{c}0.006 \\
(0.005)\end{array}$ & -30.02 & 62.05 & 66.08 & 20 & 11 & 416 \\
\hline $\begin{array}{c}\text { Número de acuerdos comer- } \\
\text { ciales en vigor }\end{array}$ & $1.76^{* * *}$ & $\begin{array}{c}0.57^{* * *} \\
(0.112)\end{array}$ & -23.00 & 48.00 & 52.06 & 20 & 11 & 425 \\
\hline
\end{tabular}

${ }^{*} p<0.05 ;{ }^{* *} p>0.01 ; * * * p<0.000$

Fuente: Elaboración propia. 